\title{
Spatiotemporal Neurodynamics Underlying Internally and Externally Driven Temporal Prediction: A High Spatial Resolution ERP Study
}

\author{
Giovanni Mento, Vincenza Tarantino, Antonino Vallesi, \\ and Patrizia Silvia Bisiacchi
}

\begin{abstract}
Temporal prediction (TP) is a flexible and dynamic cognitive ability. Depending on the internal or external nature of information exploited to generate TP, distinct cognitive and brain mechanisms are engaged with the same final goal of reducing uncertainty about the future. In this study, we investigated the specific brain mechanisms involved in internally and externally driven TP. To this end, we employed an experimental paradigm purposely designed to elicit and compare externally and internally driven TP and a combined approach based on the appli-
\end{abstract}

\section{INTRODUCTION}

Temporal prediction (TP) is a fundamental ability for life because it allows reducing uncertainty about the future and optimizing our behavior in reaction to upcoming events (Nobre, Correa, \& Coull, 2007). Crossing the street at the right moment or guessing the starter's shot in a 100-m competition are just two real-life examples of it. TP can rely upon both external and internal sources of information. Externally driven TP (E-TP) refers to the ability of preallocating cognitive resources in a precise point in time according to environmental cues, like a temporally regular structure (Barnes \& Jones, 2000) or a signal providing predictive information about the onset of a task-relevant stimulus, a phenomenon also known as temporal orienting (TO; Coull, 2009; Correa \& Nobre, 2008; Nobre, 2001). In contrast, internally driven TP (I-TP) exploits the unidirectional elapsing of time itself intrinsically and conditionally biasing target predictability (Luce, 1986; Drazin, 1961; Karlin, 1958). A classic example of I-TP is the well-known variable foreperiod (FP) effect (Vallesi, 2010; Niemi \& Näätänen, 1981; Woodrow, 1914), consisting of faster detection and discrimination responses as temporal variability and uncertainty between events decrease. Both TO and FP are known to speed up motor detection and to enhance perceptual processing of targets occurring at the predicted than unpredicted or invalidly predicted moment (Coull, 2009). Neuroimaging

University of Padua cation of a distributed source reconstruction modeling on a high spatial resolution electrophysiological data array. Specific spatiotemporal ERP signatures were identified, with significant modulation of contingent negative variation and frontal late sustained positivity in external and internal TP contexts, respectively. These different electrophysiological patterns were supported by the engagement of distinct neural networks, including a left sensorimotor and a prefrontal circuit for externally and internally driven $\mathrm{TP}$, respectively.

studies using fMRI have provided consistent evidence that E-TP entails a left sensorimotor cortical circuit, primarily including inferior parietal (IPC), premotor (PMA), and supplementary motor (SMA) areas (Coull, Davranche, Nazarian, \& Vidal, 2013; Cotti, Rohenkohl, Stokes, Nobre, \& Coull, 2011; Davranche, Nazarian, Vidal, \& Coull, 2011; Wiener, Turkeltaub, \& Coslett, 2010; Cui, Stetson, Montague, \& Eagleman, 2009; Macar, Coull, \& Vidal, 2006; Field \& Wann, 2005; Dreher, Koechlin, Ali, \& Grafman, 2002; Coull, Frith, Büchel, \& Nobre, 2000; Sakai et al., 2000; Coull \& Nobre, 1998). With regard to I-TP, a pivotal role has been hypothesized for the right lateral pFCs (Vallesi, McIntosh, Shallice, \& Stuss, 2009; Vallesi, Shallice, \& Walsh, 2007; Vallesi, Mussoni, et al., 2007; Stuss et al., 2005; BischoffGrethe, Martin, Mao, \& Berns, 2001; Henson, Shallice, \& Dolan, 1999) that may be involved in updating the conditional probability of stimulus occurrence over time, compatibly with a role of this region in monitoring (Petrides, 2005). However, this distinction may sound too naive if translated into everyday life. Because the passage of time is a pervasive, unidirectional, and unavoidable aspect of our life, it may bias the subjective conditional probability of the occurrence of events regardless of the presence of additional TO mechanisms. Moreover, although several studies showed "where" E-TP and I-TP are differently grounded in the brain, one pivotal, still unsolved, issue concerns "when" such networks are instantiated and "how" they interplay in relation to target predictability changing over time. This is mostly because fMRI provides low resolution in depicting the temporal dynamics of the 
neural activity. Some studies have attempted to fill this gap using ERPs that are featured by an excellent temporal resolution. It has been reported that TP elicits pretarget neural activity (Capizzi, Correa, \& Sanabria, 2013; Correa, Lupiáñez, \& Tudela, 2006; Los \& Heslenfeld, 2005; Miniussi, Wilding, Coull, \& Nobre, 1999). In particular, the contingent negative variation (CNV) has been proposed to be a reliable hallmark of temporal anticipatory processes (Kononowicz \& van Rijn, 2011, 2014; Mento, 2013; Mento, Tarantino, Sarlo, \& Bisiacchi, 2013; Van Rijn, Kononowicz, Meck, Ng, \& Penney, 2011). However, the questions of how the two distinct neural networks underlying E-TP and I-TP are differently and progressively engaged over time as well as whether such networks translate into same or different scalp-level electrophysiological patterns still remain unanswered. In other words, although the "where" and "when" aspects of TP instantiation in the brain are two complementary sides of the same coin, an attempt to make them converge toward a unitary framework to explain how the brain generates prediction about the future is lacking.

In this study, we investigated the spatiotemporal neurodynamics of different types of TP. In particular, we designed an experimental paradigm, in which TO and FP tasks were factorially combined to generate E-TP and I-TP, respectively. Using a combined approach based on the application of a distributed source reconstruction modeling on a high-resolution EEG array (Michel \& Murray, 2012), we were able to depict and compare the time course of both the scalp electrical activity and the underlying putative cortical sources of different types of TP.

\section{METHODS}

\section{Participants}

Nineteen healthy, right-handed volunteers (mean age $=$ $24.4 \pm 2.5$ years) with normal or corrected-to-normal vision participated in the experiment. All volunteers received course credits for their participation in the study and provided informed written consent to the study protocol, which had been approved by the ethical committee of the School of Psychology of the University of Padua (Protocol No. 1281) and was conducted according to the principles expressed in the Declaration of Helsinki.

\section{Experimental Procedure and Stimuli}

The experiment was conducted in a dimly illuminated and electrically shielded room. Stimuli were presented on a 19-in. monitor at a resolution of $1280 \times 1024$ pixels. Participants were comfortably seated in a chair at a viewing distance of $100 \mathrm{~cm}$ from the monitor. All participants performed a prediction task adapted from Coull et al. (2013).

\section{Experimental Task}

Each trial began with the display of a visual cue (500 msec), followed by the presentation of a brief target stimulus (130 msec) after a variable interval. The SOA between cue and target could last either 1100 or 1900 msec. All visual stimuli were white, centrally presented on a gray background. The visual cue consisted of two concentric circles, and the target stimulus comprised two horizontally aligned crosses. Participants were instructed to detect targets by pressing a button of the response box with the right index finger as quickly as possible. The intertrial interval was randomly manipulated between 600 and $1500 \mathrm{msec}$. The informative value of the cue was manipulated, leading to different TP conditions as detailed below.

\section{E-TP Condition}

In the E-TP condition (Figure 1A), the visual cue provided fixed temporal information concerning the SOA duration (temporal cue). In particular, either the inner or outer circle of the cue could be highlighted, generating a temporal short (T-Short) or long (T-Long) SOA condition, with durations of 1100 and $1900 \mathrm{msec}$, respectively. In line with prior studies (Correa et al., 2006; Miniussi et al., 1999; Coull \& Nobre, 1998), the temporal cueing should induce participants to generate TO, leading to faster RTs for temporally predictable targets. Cue validity was set up at $100 \%$ of trials and no catch trials were delivered; therefore, the cue always predicted a short or a long SOA. This was performed to exacerbate the likelihood of TO effect for both short and long intervals. The association between the inner or outer part of the circle and the SOA duration was counterbalanced between participants. At cue offset, the screen blanked out, and the target stimulus always appeared after the cued interval. Furthermore, participants made a speeded button press to the appearance of this stimulus.

\section{I-TP Condition}

In the I-TP (Figure 1B), both inner and outer circles of the cue were highlighted, providing no temporally precise information about SOA duration (neutral cue). Consistent with the FP paradigm, in this case, the cue simply acted as a warning signal, which prepared for the forthcoming target onset without furnishing temporal information about it. Nevertheless, for the E-TP condition, SOA was also manipulated to create neutral short (N-Short) and long (N-Long) trials, with the same durations as those for temporal cueing condition (1100 and $1900 \mathrm{msec}$, respectively). To maximize the FP effect, we used an "aging" probability distribution (Niemi \& Näätänen, 1981), with an equal a priori odd ratio for each SOA duration. This 
Figure 1. Experimental task. Participants had to produce speeded target detections by pressing a button of the response box. Targets could be either temporally (A) or neutrally (B) cued, inducing E-TP or I-TP, respectively. As well, SOA could have short (1100 msec) or long (1900 msec) duration. (C) Experimental design. Both CUE and SOA factors were manipulated leading to the following comparisons:

(1) T-Short versus N-Short (E-TP short SOA); (2) T-Long versus N-Long (E-TP long SOA); (3) T-Short versus T-Long (E/I-TP); (4) N-Short versus N-Long (I-TP). (D) Hypothetical graphical representation of target predictability over time: Consistently with previous research (Coull et al., 2013), we expected target predictability to unfold differently for short and long SOA and for E-TP and I-TP.

More in detail, we hypothesized E-TP to be maximum in the short-range temporal window, resulting in higher target predictability for temporally than neutrally cued targets at short SOA. However, we also expected this effect to vanish at long SOA, due to the passage of time generating I-TP and strongly biasing target predictability. Finally, because the passage of time is unavoidably present in both E-TP and I-TP, we expect it to add predictability also for E-TP, resulting in overall faster performances for long SOA, independent from TO. (E) High-density scalp sensors used in the experiment. The colors represent the different scalp-ROIs created by clustering together groups of adjacent sensors.

probability distribution is known to obey a hazard function (Woodrow, 1914). This consists of the progressive probability redistribution a posteriori as a simple function of the passage of time. In fact, the subjective probability of target onset will increase over time given that it has not yet occurred.

\section{Experimental Design}

The experimental manipulation yielded a factorial $2 \times$ 2 design, with cue (Temporal vs. Neutral) and SOA (Short vs. Long) as experimental factors. The pairwise comparisons among all conditions allowed to test for the distinct and common effects of E-TP and I-TP conditions. As shown in Figure 1C, E-TP was measured by contrasting temporal vs. neutral trials separately for short (E-TP short SOA) and long (E-TP long SOA) intervals, whereas I-TP was measured by contrasting neutral short vs. neutral long trials. Furthermore, contrasting temporal short vs. temporal long trials (E/I-TP) allowed to specifically measure the interaction between E-TP and I-TP. In fact, in E-I/ $\mathrm{TP}$, performance of participants is supposed to be biased by both E-TP (as targets are here always predictable) and I-TP (as in long SOA trials should anyway induce faster RTs). All conditions were matched for sensorimotor requirements because the sequence of stimuli and the required response were always the same, the only difference between conditions being the level of target predictability (Figure 1D).

Each participant underwent a total of four experimental blocks, including two temporal and two neutral blocks. In the two temporal blocks a total of 120 trials (60 for short and 60 for long SOA condition) were randomly delivered. In the remaining two neutral blocks, participants were given a total of 120 trials (60 for short and 60 for long SOA condition) that were randomly delivered. The experiment was randomized block-wise. The order of the blocks was counterbalanced between participants. E-Prime 2 software (Psychology Software Tools, Pittsburgh, PA) was used to create and administer the stimuli.

\section{EEG Recordings}

During the entire task, the EEG was continuously recorded and amplified using a geodesic high-density EEG system (EGI GES-300), through a precabled 128-channel HydroCel Geodesic Sensor Net (HCGSN-128) and referenced to the vertex. The sampling rate was $500 \mathrm{~Hz}$. The impedance was maintained below $30 \mathrm{~K} \Omega$ for each sensor. To reduce the presence of EOG artifacts, participants were instructed to limit both eyeblinks and eye movements as much as possible. 


\section{Data Analysis}

\section{Behavioral Analysis}

RTs to target stimuli in all experimental conditions were recorded. RTs below $150 \mathrm{msec}$ were not considered. A $2 \times 2$ repeated-measures ANOVA was performed, with CUE (Temporal vs. Neutral) and SOA (Short vs. Long) as within-subject factors. The Bonferroni post hoc test was used to correct for multiple comparisons. Effect size was calculated by using the partial eta square $\left(\eta_{p}^{2}\right)$.

\section{ERP Analysis}

ERPs were extracted from the EEG signal using Net Station 4.4.1 software (EGI Instruments, Eugene, OR). The EEG signal was segmented offline into epochs starting $200 \mathrm{msec}$ before cue onset and ending $2200 \mathrm{msec}$ after. Epochs were $20 \mathrm{~Hz}$ digitally low-pass filtered and automatically processed to mark bad channels, eyeblinks, and eye movements. Channels with $>20 \%$ of rejected trials were marked as bad and interpolated with the surrounding electrodes. All the trials marked as bad were visually inspected and, if containing artifacts, were rejected. The remaining epochs contaminated by eyeblinks were corrected using Gratton's algorithm (Gratton, Coles, \& Donchin, 1983) and re-referenced to the average of all electrodes. The artifact-free trials were then averaged for each participant, separately for each condition. The signal was aligned to the baseline by subtracting the mean signal amplitude in the prestimulus interval. Two-dimensional reconstructions of scalp voltages were computed using a high-density, spherical spline interpolation map for each condition. ERP amplitudes were analyzed by pooling the values of six neighboring electrodes in nine ROIs (scalp-ROIs) covering evenly the scalp surface. As shown in Figure 1E, the following nine scalp-ROIs were considered: anterior left $(\mathrm{AL})$, midline (AM), and right (AR); central left (CL), midline $(\mathrm{CM})$, and right $(\mathrm{CR})$; posterior left $(\mathrm{PL})$, midline $(\mathrm{PM})$, and right (PR). The mean ERP voltage amplitude values of 200-msec interval windows from 0 to $2200 \mathrm{msec}$ from cue onset were calculated to allow coverage of the whole cue-target epoch for all conditions, without selecting a priori wave-related interval windows. Statistical analyses were restricted to specific consecutive 200-msec temporal windows postcue. Namely, for short epochs (T-Short and N-Short), we divided the postcue interval in three consecutive temporal windows covering the interval between 600-800 (T1 bin), 800-1000 (T2 bin), and 10001200 (T3 bin) msec from cue onset. In contrast, for long epochs (T-Long and N-Long), we focused on a later temporal window covering the intervals between 1400-1600 (T4 bin), 1600-1800 (T5 bin), and 1800-2000 (T6 bin) msec from cue onset. ERP mean amplitudes were then entered in separated three-way ANOVA models, depending on the considered comparison. The effect of the following within-subject factors was analyzed:
CUE, scalp-ROI, and temporal BIN, leading to a $2 \times 9 \times$ 3 repeated-measure ANOVA. The temporal BIN factor varied depending on the epoch length considered. More specifically, T1, T2, and T3 bins were considered when testing short-interval conditions. Instead, T4, T5, and T6 bins were considered when testing long-interval conditions. Furthermore, planned paired $t$ test comparisons were performed to compare different temporal bins between different conditions. The following comparisons were made: $\mathrm{T} 1$ vs. T4, T2 vs. $\mathrm{T} 5$, and $\mathrm{T} 3$ vs. T6. A Bonferroni post hoc correction was used to test for significant differences in ERP amplitude. In addition, effect size was calculated by using the partial eta square $\left(\eta^{2}\right)$. Finally, Pearson correlations were independently conducted between ERP amplitudes and RTs for short and long interval trials and for all temporal interval BINs across participants.

\section{Brain Source Analysis}

The cortical generators of cue-locked ERP activity were reconstructed. To do this, the conductive head volume was modeled according to OpenMEEG BEM (Gramfort, Papadopoulo, Olivi, \& Clerc, 2010) as implemented in the Brainstorm software package (Tadel, Baillet, Mosher, Pantazis, \& Leahy, 2011). The solution space was constrained to the cerebral cortex, which was modeled as a three-dimensional grid of 15,028 fixed dipoles normally oriented to the cortical surface. Furthermore, the inverse transformation was applied to the Montreal Neurological Institute (MNI) canonical mesh of the cortex to approximate real anatomy. The EEG sensor positions were coregistered with the default anatomical mesh by employing rigid rotations and translations of digitized landmarks (anterior and posterior commissure, interhemispheric scissure, nasion, and left and right tragus). The inverse modeling was based on minimum norm solutions (weighted minimum-norm current estimate) implemented as a routine of the Brainstorm platform. The covariance matrix was assumed to be independent across EEG sensors, with fixed variance computed from prestimulus recordings. For each participant, the sources were projected to a standard anatomical template (MNI) and their activity was transformed in absolute $Z$ scores relative to the baseline. The absolute values of the $Z$ scores were then averaged across participants into $200-\mathrm{msec}$ temporal bins, resulting on the same temporal windows used for ERP analysis. The cortical activations were located according to the anatomical atlas of Tzourio-Mazoyer (TzourioMazoyer, Landeau, Papathanassiou, et al., 2002) adapted for cortical space solution. It deals with an approximate re-projection of the volume-based Tzourio-Mazoyer atlas on a cortex surface obtained with the BrainVISA software and implemented in the Brainstorm software as a cortical localization procedure (Tadel et al., 2011). 


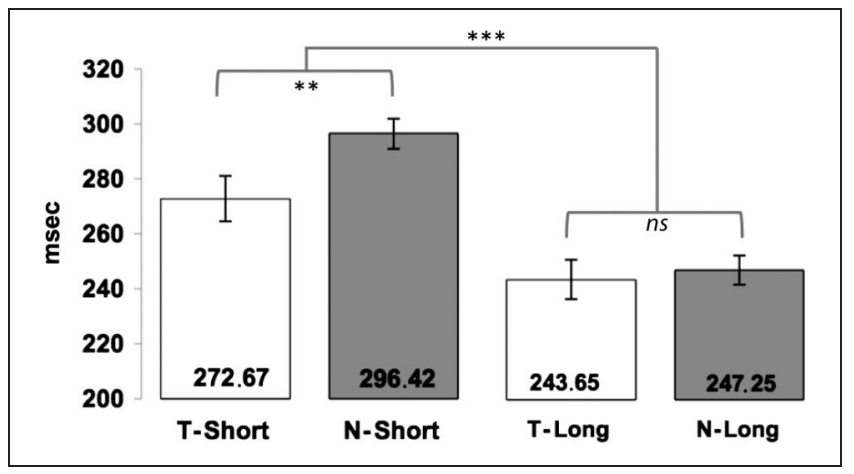

Figure 2. Behavioral performance. RTs obtained for T-Short, $\mathrm{N}$-Short, T-Long, and N-Long conditions. Error bars indicate standard errors of the mean. Overall, participants were faster for temporally than neutrally cued targets and for long than for short SOAs. However, the CUE type interacted with SOA, because at short SOAs participants benefitted from the temporal information provided by the CUE, whereas at long SOAs their performance was not affected by CUE type. $* p<.05 ; * * p<.01 ; * * p<.001 ; n s=$ not significant.
To examine the cortical regions that were significantly activated at the moment of maximum expectancy in each condition, we statistically compared source activation values of the temporal bins immediately preceding target onset for short and long cues (T3 and T6, respectively), performing two separate $t$ tests. Thresholding on the size of the effects was applied: only clusters of at least 10 cortical vertices in the distributed sources model were considered. Then, the source map vertices where the $t$ statistics exceeded a critical value $(p<.05$, FDRcorrected for multiple comparisons) were clustered into cortical ROIs based on their adjacency across the two dimensional cortical sheet. Only the cortical ROIs significantly activated were reported and identified according to the MNI coordinate system. Cortical map activations and statistics have been separately reported for short and long SOA. To more accurately depict the time course of the activation of the main cortical ROIs identified, we used the scout analysis tool in Brainstorm. This procedure allows one to cluster subsets of neighboring vertices and to plot their activation values for the temporal dimension.

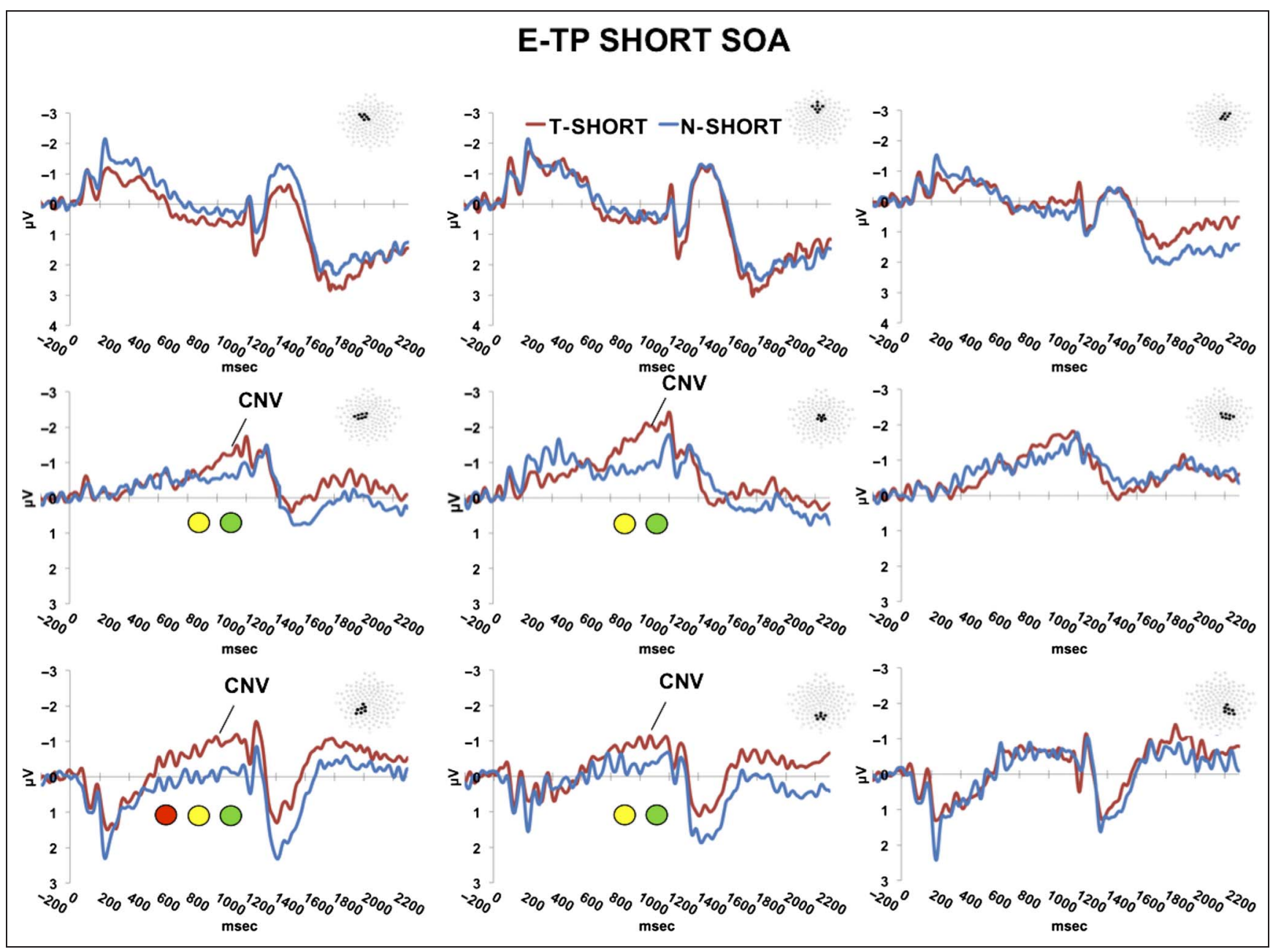

Figure 3. E-TP short SOA. ERPs elicited by temporal (red continuous lines) and neutral (blue continuous lines) cues in nine scalp-ROIs. Colored circles represent the temporal bins showing significant amplitude differences. 


\section{RESULTS}

\section{Behavioral Data}

Both E-TP and I-TP induced significant effects on participants' behavioral performances. As shown in Figure 2, participants were overall faster not only when targets were temporally predictable (main effect of the CUE; $F(1$, 18) $=8.66 ; p<.01 ; \eta_{p}^{2}=.32$ ) but also when they were preceded by long than short SOA (main effect of the SOA; $\left.F(1,18)=79.92 ; p<.0001 ; \eta_{\mathrm{p}}^{2}=.81\right)$. In addition, a significant CUE $\times$ SOA interaction $(F(1,18)=8.84 ; p<$ $\left..001 ; \eta_{\mathrm{p}}^{2}=.32\right)$ revealed that E-TP produced an asymmetrical effect, as the speeding up of RTs induced by the temporal cue was evident for short $(p<.005)$ but not long $(p>.4)$ SOAs. The Bonferroni post hoc tests also confirmed the presence of faster RTs for the T-Long than T-Short condition $(p<.001)$ and also that RTs were overall faster in the E-TP than in the I-TP condition $(p<.01)$.

\section{ERP Data}

Data from three participants were discarded for excessive EEG artifacts. In the remaining participants, a mean of $11 \pm 1 \%$ epochs were rejected. There were no significant differences $(p>.7)$ between the percentage of epochs rejected in all conditions.

\section{$E-T P$}

The visual inspection of the electrophysiological results globally revealed larger ERP negative activity following temporal cues spreading over central and posterior scalp sites (Figure 3). This negativity consisted of a CNV arising just after the end of the cue-locked sensory evoked response and peaking in correspondence to target onset. ANOVA confirmed this pattern revealing a main effect of CUE $\left(F(1,15)=6.34 ; p<.03 ; \eta_{\mathrm{p}}^{2}=.29\right)$, with temporal cue overall producing more negative ERP activity

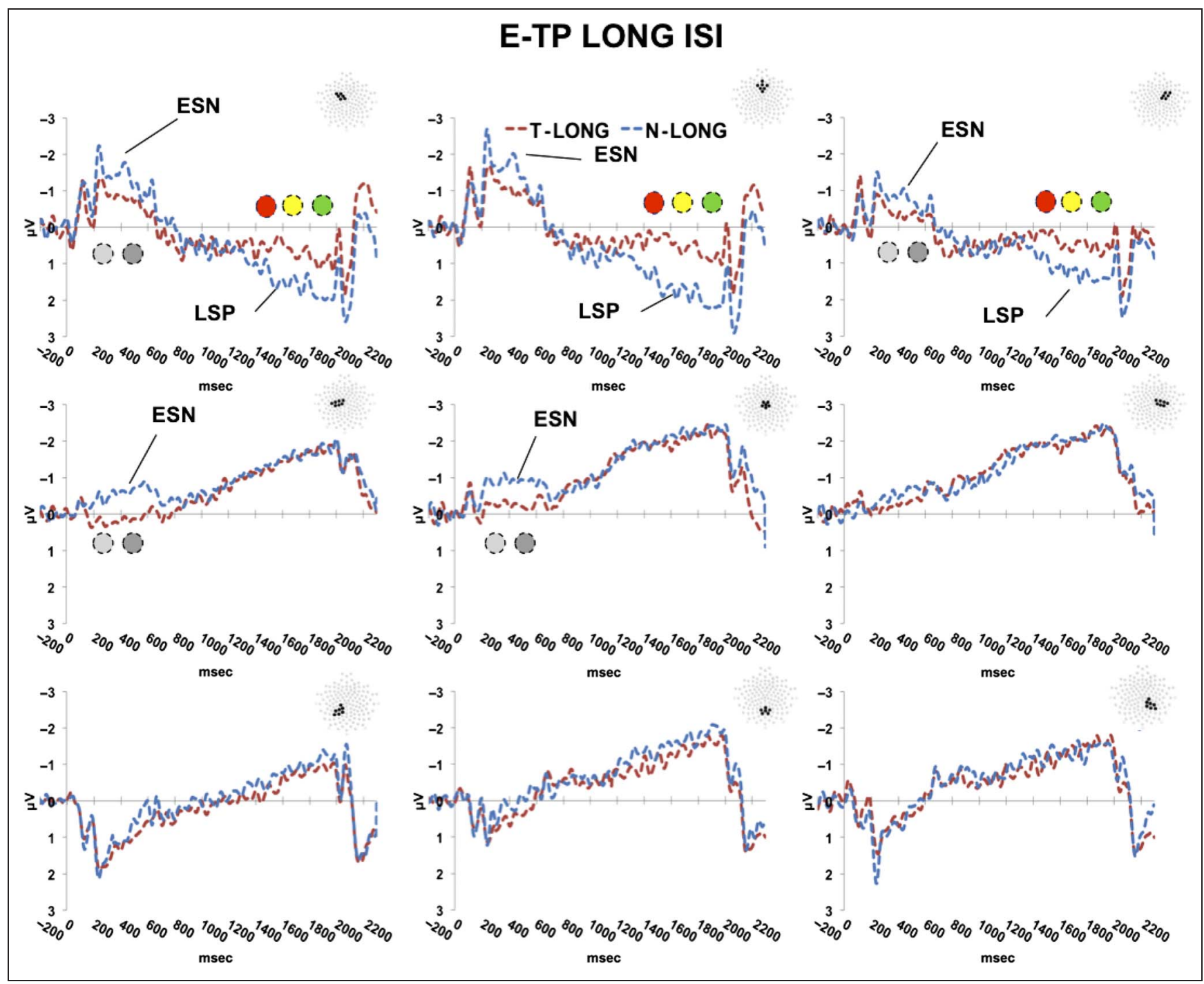

Figure 4. E-TP long SOA. ERPs elicited by temporal (red dashed lines) and neutral (blue dashed lines) cues in nine scalp-ROIs. Colored dashed circles represent the temporal bins showing significant amplitude differences. 


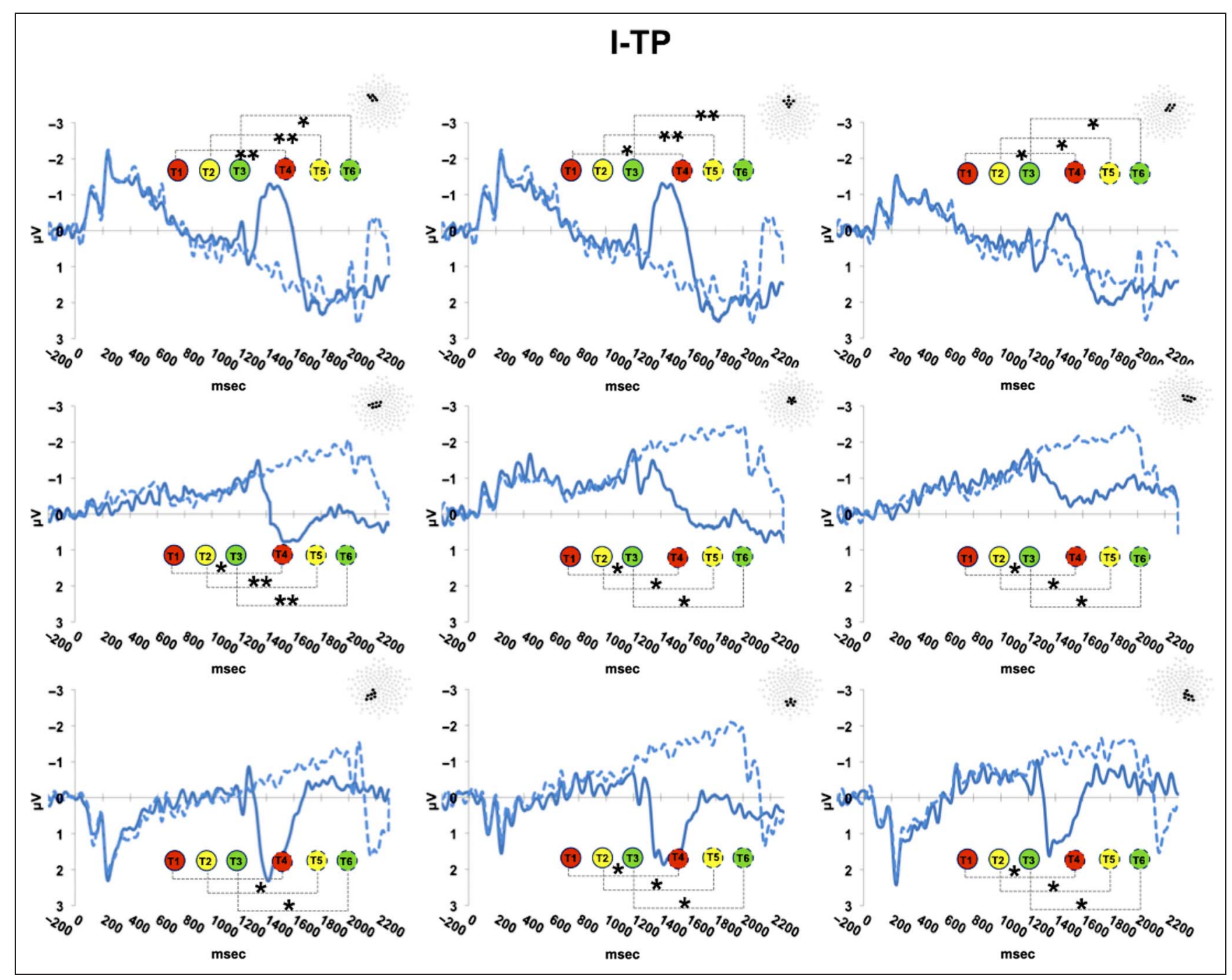

Figure 5. I-TP. ERPs elicited for neutral short (blue continuous lines) and long (blue dashed lines) trials. No amplitude differences were found when comparing the same temporal bins. Horizontal dashed lines represent significant amplitude differences $(* p<.05$ and $* * p<.01)$ between different bins.

than neutral cue. Furthermore, a CUE $\times$ ROI $\times$ BIN interaction $\left(F(16,240)=4.26 ; p<.001 ; \eta_{p}^{2}=.22\right)$ revealed that this effect was stronger (i.e., larger $\mathrm{CNV}$ ) at $\mathrm{CL}, \mathrm{CM}$, $\mathrm{PL}$, and PM scalp-ROIs and only in T2 and T3 bins (all ps < .05). An earlier cue effect was detected at PL Scalp-ROI, with CNV differentiating between conditions already at T1 bin $(p<.02)$.

Concerning long SOAs (Figure 4), the waveform visual inspection revealed the presence of a CNV arising soon after the sensory potentials evoked by cue onset and peaking in correspondence to target onset, in central and posterior sites. Unlike the pattern observed at short SOAs, no cue-related CNV differences in any scalp-ROIs was observed; however, a larger late sustained positivity (LSP) was elicited by neutral cues in frontal sites. This asymmetrical pattern was supported by a significant two-way CUE $\times$ ROI interaction $(F(8,120)=5.39 ; p<$ $\left..001 ; \eta_{\mathrm{p}}^{2}=.26\right)$, with $\mathrm{N}$-Long eliciting larger LSPs in frontal scalp-ROI (AL, AM, and AR) for all considered tem- poral bins (T4, T5, and T6; all $p s<.03$ ). An additional larger early sustained negativity (ESN) for the N-Long condition at frontocentral sites between approximately 200 and $600 \mathrm{msec}$ from cue onset emerged. To statistically test this difference, the mean ERP amplitude of two additional 200-msec temporal bins, that is, from 200 to $400 \mathrm{msec}$ (Ta bin) and from 400 to $600 \mathrm{msec}$ (Tb bin), were extracted. Furthermore, we performed a $2(\mathrm{CUE}) \times 9(\mathrm{ROI}) \times 2(\mathrm{Ta}$ vs. Tb) repeated-measures ANOVA that yielded a CUE $\times$ ROI interaction $(F(8,120)=$ 3.49; $\left.p<.005 ; \eta_{\mathrm{p}}^{2}=.18\right)$. Post hoc tests further confirmed that the $\mathrm{ESN}$ was larger at $\mathrm{AL}(p<.01), \mathrm{AR}(p<$ $.05)$, CL $(p<.05)$, and marginally at CM $(p=.06)$ scalpROIs in both $\mathrm{Ta}$ and $\mathrm{Tb}$ bins.

\section{I-TP}

As shown in Figure 5, no waveform differences were appreciable in ERP activity at T1, T2, and T3 bins (all 
$p s>.4)$. In effect, this was an expected finding given that target occurrence was actually unpredictable until the end of the first useful interval (i.e., the short SOA). Hence, there was no reason to observe postcue differences within the first $1100 \mathrm{msec}$ after cue onset. However, planned paired $t$ test comparisons between different temporal windows revealed that, following the end of the short SOA, all later bins (i.e., T4, T5, and T6) showed larger positive activity at frontal (all $t$ s $<$ -2.3 ; all $p s<.05)$ and larger negative activity at central (all $t \mathrm{~s}>2.4$; all $p \mathrm{~s}<.05$ ) and posterior (all $t \mathrm{~s}>2.2$; all ps $<.05)$ scalp-ROIs.

\section{E/I-TP}

As stated above, the E/I-TP effect refers to the combined effect of both E-TP and I-TP. In such cases, ERP correlates should reflect the effect of SOA lengthening (i.e., I-TP) but in a temporally certain context (E-TP), given that participants were equally allowed to predict target occurrence at short and long SOAs. As displayed in Figure 6, a waveform pattern similar to that detected for E-TP at short SOA (Figure 3) was observed. This consisted of larger CNV at central and posterior sites for the T-Short than N-Short condition, confirmed by significant CUE $\times$ ROI interaction $\left(F(8,120)=2.13 ; p<.05 ; \eta_{\mathrm{p}}^{2}=.12\right)$. Post hoc tests revealed a larger CNV for the T-Short than T-Long condition only at central (CL, CM) and posterior (PL and PM) scalp-ROIs (all $p s<.05$ ) for all temporal bins. Moreover, planned paired $t$ test comparisons revealed a significant difference in amplitude when examining the early portion of the CNV between short and long epochs (T2 vs. T5 bins) at CL $(t(15)=2.15 ; p<.05)$, $\mathrm{CM}(t(15)=3.9 ; p<.002)$, and CR $(t(15)=2.83 ; p<.02)$, as well as the late portion of the CNV (T3 vs. T6 bins) at CL $(t(15)=2.31 ; p<.05), \mathrm{CM}(t(15)=2.13 ; p<.05)$, and CR $(t(15)=2.24 ; p<.05)$.

\section{Correlation between RTs and ERP Amplitude}

RTs were significantly correlated to ERP amplitude measured in proximity of target onset (i.e., T3 and T6 bins for

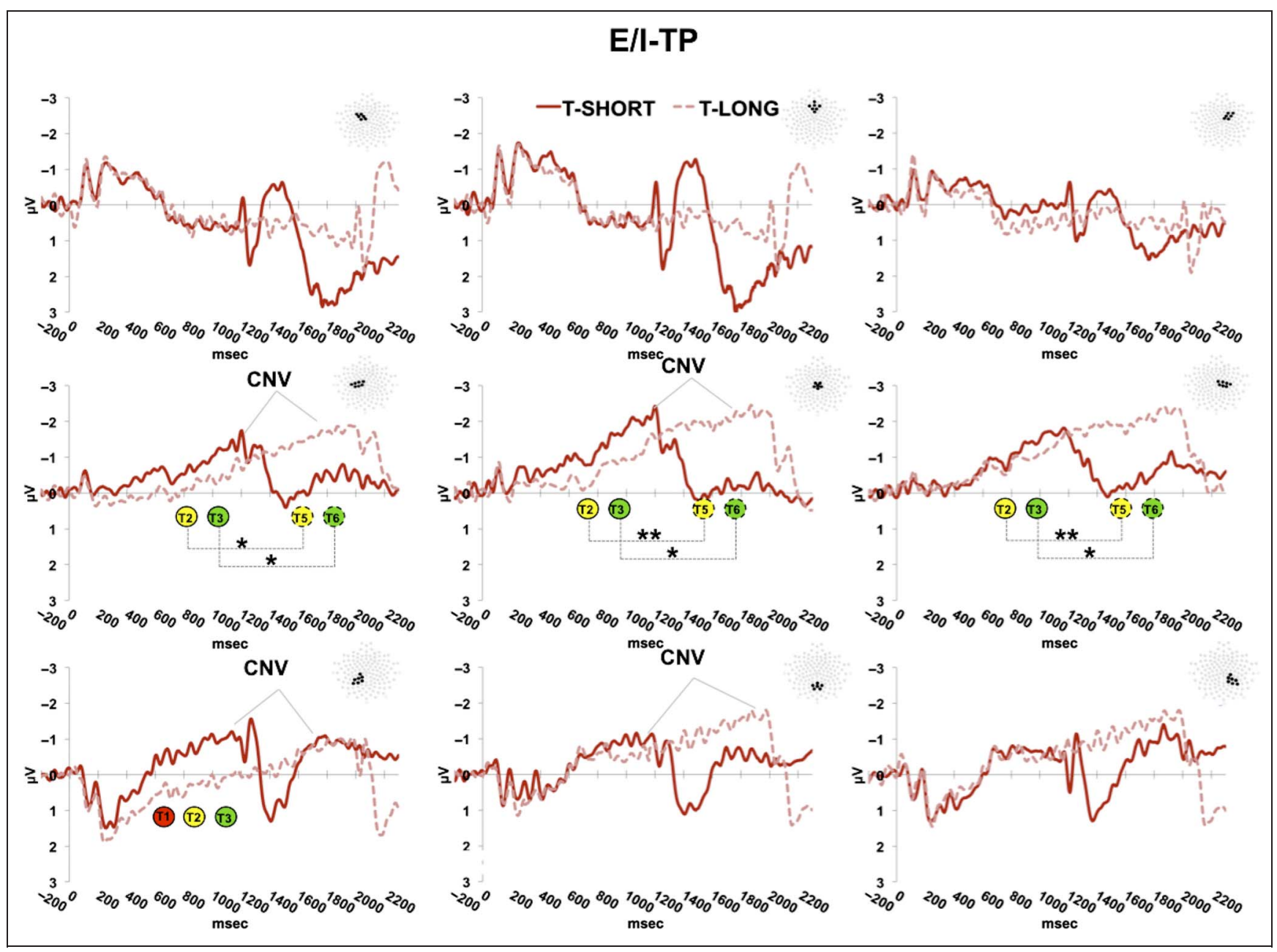

Figure 6. E/I-TP. ERPs elicited by temporal short (red continuous lines) and long (red dashed lines) trials in nine scalp-ROIs. Colored circles represent the interval bins showing significant amplitude differences. Horizontal dashed lines represent significant amplitude differences $(* p<.05$ and $* * p<.01)$ between different bins. 


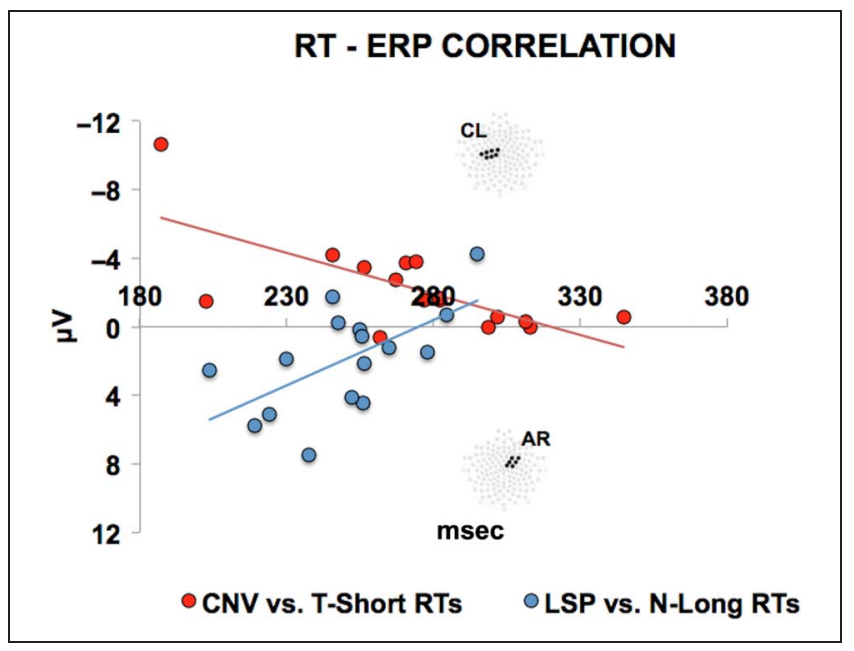

Figure 7. RT-ERP correlation. Linear correlations between RTs and mean ERP amplitude at different scalp-ROIs. Top half-side: correlation between CNV amplitude and RTs relatively to T-Short condition. The CNV amplitude of single participants (red dots) significantly predicts RTs. Bottom half-side: correlation between LSD amplitude and RTs relatively to N-Long condition. The LSD amplitude of single participant (blue dots) significantly predicts RTs.

short and long epochs, respectively). More specifically, in the T-Short condition RTs were directly correlated to CNV amplitude at CL $(r=.65 ; p<.01), \mathrm{CM}(r=.69$; $p<.005)$, PL $(r=.62 ; p<.02)$, and PM $(r=.68 ; p<.02)$ scalp-ROIs. In the N-Long condition, RTs were inversely correlated to LSP amplitude at AL $(r=-.6 ; p<.02)$ and AR $(r=-.57 ; p<.02)$ but not to central and posterior $\mathrm{CNV}$ amplitude (all $p \mathrm{~s}>.2$ ). In other words, when targets were predictable as occurring at short intervals (E-TP short SOA), participants' performance was explained by left centro-posterior CNV. However, when targets were unpredictable (I-TP) but actually occurred at long SOA, participants' performance was correlated by bilateral frontal LSP amplitude (Figure 7).

\section{Brain Source Analysis}

The reconstruction of the cortical sources for short and long SOA conditions at the moment of maximum target predictability (T3 and T6 for short and long trials, respectively) is shown in Figure 8, together with ERP and scalp maps. Overall, a distributed neural network was identified, mainly including frontoparietal cortical regions. In particular, in the temporal conditions (T-Short and T-Long) a sensorimotor network was engaged soon after cue offset. This mostly included l-IPC (BA 39), 1-PMA (BA 6), and SMA (BA 6) bilaterally. By contrast, in the neutral conditions (N-Short and N-Long), a prevalently frontal network was recruited, mainly including $\mathrm{pFC}$ (BA 46) and the SMA.

Globally, the cortical source reconstruction draws a clear-cut picture of the spatiotemporal dynamics of distinct two neural networks selectively recruited for dif- ferent types of TP. These relied on a prevalently left sensorimotor circuit for externally driven conditions and on a prevalently right frontal circuit for I-TP. Interestingly, at long SOAs, the temporal condition revealed a similar pattern as that for short SOAs, although it was featured by an additional late activation of the r-pFC.

Statistical analyses separately performed on source maps for short and long trials at the moment of maximum target predictability confirmed the specific cortical patterns elicited by temporal versus neutral cues and vice versa (Figure 9A). For short SOAs, we observed a significant activity in parietal (1-IPC), frontal (1- and r-MA; 1 - and r-PMA), and prefrontal (1-pFC and r-pFC) cortices. For long SOAs, a similar frontoparietal cortical network was observed, including nearly the same areas. However, in this case additional areas were detected, including the SMA bilaterally, the right temporal cortex (r-I/STG), and the left occipital cortex (l-V2). Noticeably, the SMA was located on the dorsal portion of the superior frontal gyrus according to the Tzourio-Mazoyer atlas (TzourioMazoyer et al., 2002) adapted for cortical space solution and implemented as a cortical atlas in the Brainstorm software. The fact that we found only the dorsal rather than mesial part of the SMA to be activated may be because of the dipole localization algorithm used to reconstruct cortical sources, which may have biased active cortical vertices toward more superficial than deep cortical areas. Hence, we cannot exclude that a more mesial portion of the SMA is involved, as previously reported by fMRI studies. Figure 9B displays the time course comparison of the cortical ROIs identified by statistical comparison and showing the strongest statistical effect for both short and long SOA.

\section{DISCUSSION}

In this study, we investigated both the behavioral correlates and spatiotemporal neurodynamics underlying E-TP and I-TP. Overall, results suggest that such processes are distinguishable and flexibly interact within the same experimental context. In terms of RTs, in the externally driven condition (E-TP), participants benefitted (i.e., showed shorter RTs) from the presence of a cue providing information about the timing of target occurrence. Similarly, in the internally driven condition (I-TP), the expectation of the target intrinsically related to the simple passage of time conditionally heightened the probability of its occurrence and determined faster responses in long SOA trials. We found that E-TP and I-TP processes interact when factorially combined (E/I-TP condition). Participants' performance benefitted more from the temporal cue in T-Long compared with that in the T-Short trials. In such trials, in addition to the intervention of external orienting mechanisms, participants internally generated higher TP as SOA increased; therefore, their RTs were shorter. This finding represents a demonstration of the 
dynamic nature of TP and is in line with the study by Coull et al. (2013), from which the experimental paradigm was adapted. The specific benefit of long SOA trials is explained by the hazard function (Coull et al., 2013; Nobre et al., 2007; Miniussi et al., 1999; Niemi \& Näätänen, 1981), which is defined as the conditional probability of an event occurring at a given time given that it has not yet occurred (Nobre et al., 2007; Luce, 1986). Unlike the study by Coull et al. (2013), we adopted an "aging" distribution (Trillenberg, Verleger, Wascher, Wauschkuhn, \& Wessel, 2000; Niemi \& Näätänen, 1981) that is an equal a priori probability for each SOA. Despite their attempt to minimize the impact of the hazard function on stimulus temporal predictability by using a "non-aging" SOA probability distribution, Coull et al. (2013) found behav- ioral performance to be still influenced by the hazard function. The aging probability distribution is known to bias the a posteriori probability of target onset, making events subjectively more predictable with time. We did not expect to find differences in participants' performance between the T-Long and N-Long conditions. Taken together, these data would suggest the existence of a hierarchical organization in TP mechanisms, with I-TP playing a highlevel role by virtue of its pervasive nature, which conditionally and unavoidably biases events' predictability over time and obeys a hazard function (Coull, 2009; Cui et al., 2009; Nobre et al., 2007). Hence, despite time elapsing per se automatically brings predictive power, the presence of external cues, which orient attention in time, further adds temporal predictability. The analysis of spatial

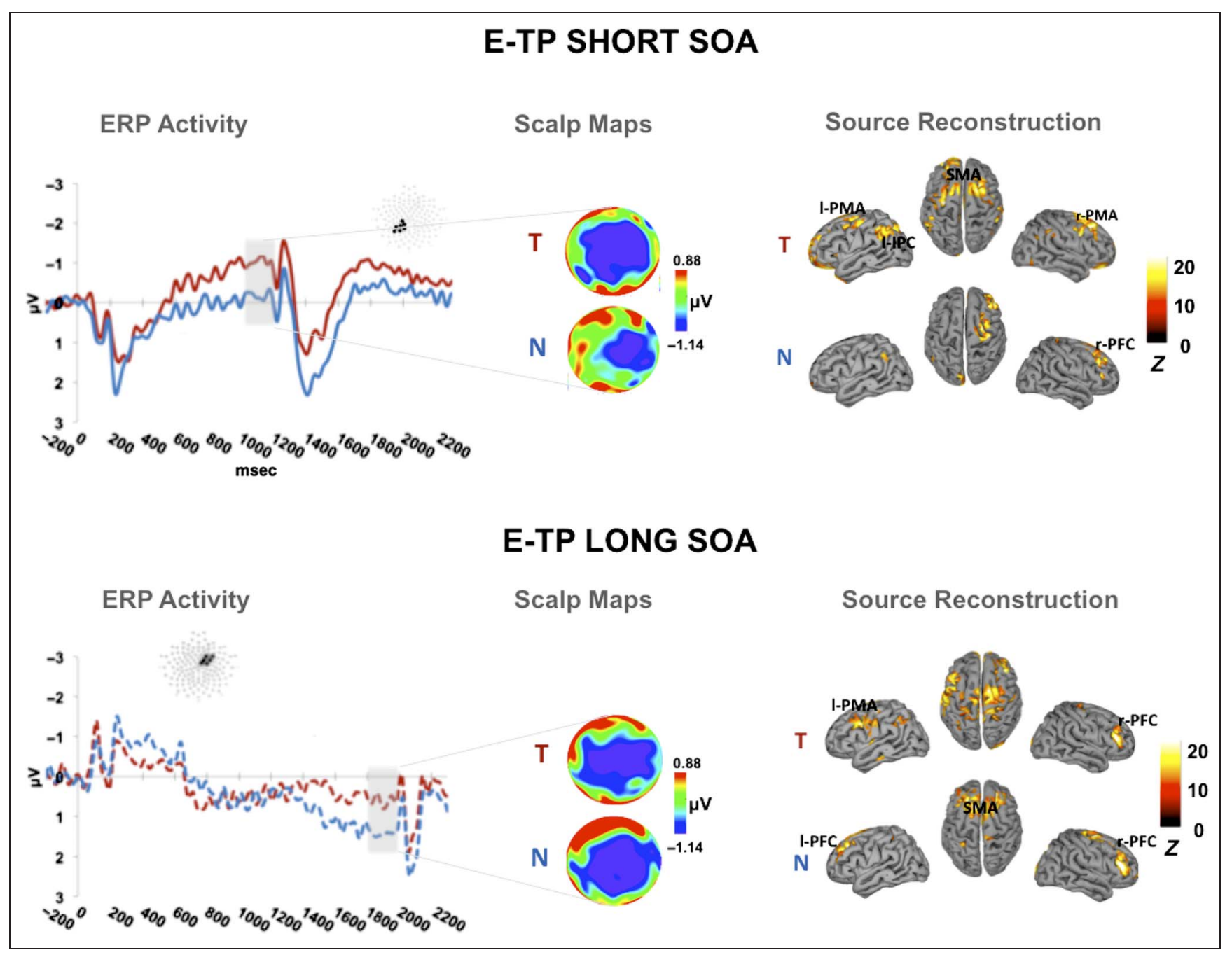

Figure 8. ERP waveforms (left column). Dissociation between E-TP short SOA (resulting in a CNV modulation) and ETP long SOA (resulting in a frontal LSP modulation). Scalp maps (central column): high-resolution averaged topographical maps covering a 200-msec postcue interval window in proximity of target onset (T3 and T6 bins for short and long SOAs, respectively). At short SOAs (top), temporal cues (T) elicited more negative scalp voltages over centro-posterior regions than neutral (N) cues. By contrast, at long SOAs (bottom) neutral cues elicited more positive scalp voltages over bilateral frontal regions than temporal cues. Brain source reconstruction (right column): at short SOAs (top) temporal cues activates a prevalently left sensorimotor cortical network, mainly including left inferior parietal (1-IPC), left premotor/motor (1-PMA/MA), and supplementary motor (SMA) areas. At long SOAs (bottom) neutral cues activate a prevalently frontal network including the SMA and the right and left pFCs (1-pFC and r-pFC). Data were adjusted using a threshold of 50\% of the maximum amplitude and a size of at least 10 vertices. 


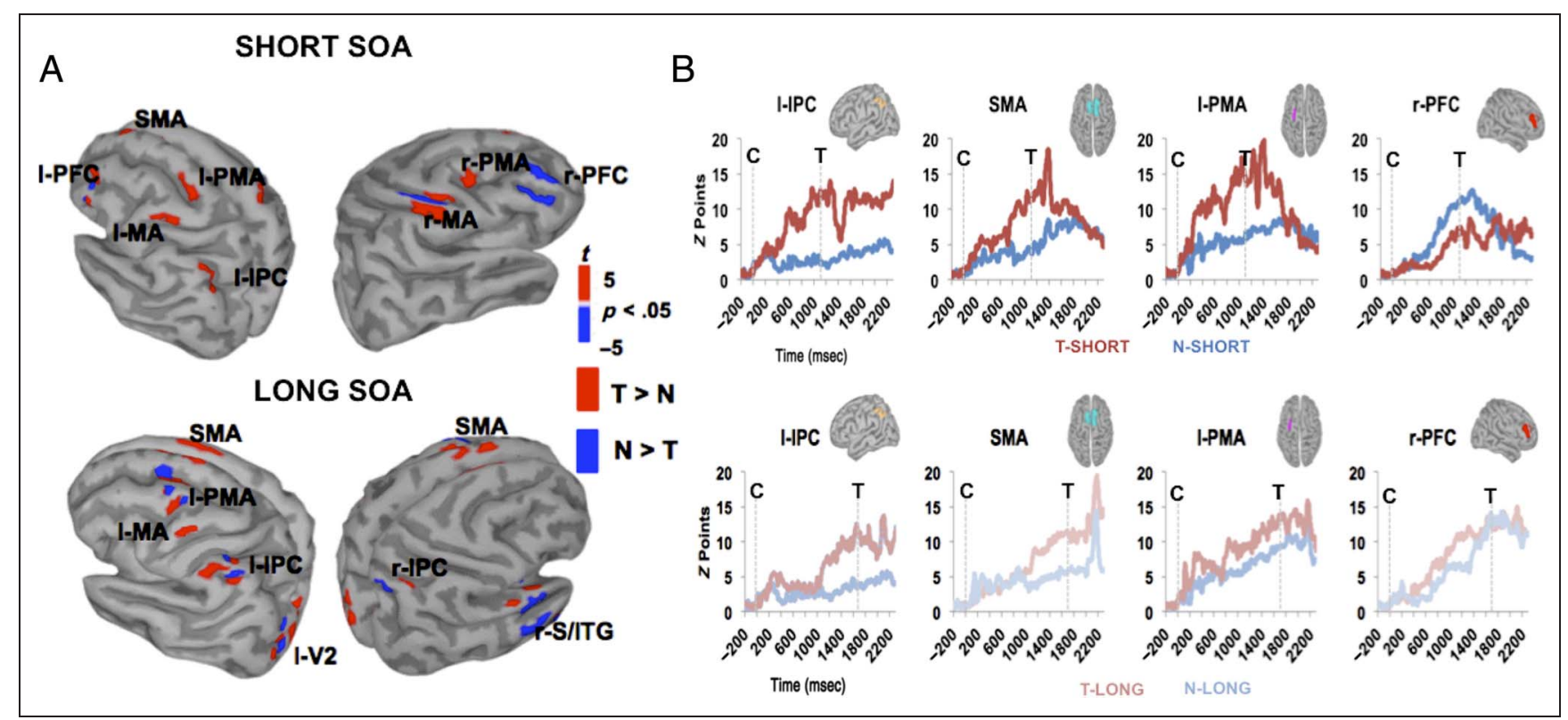

Figure 9. (A) Estimated cortical areas with significant activation. 1-IPC = left intraparietal cortex; 1-PMA = left premotor area; SMA = supplementary motor area; l-MA = left motor area; $\mathrm{r}-\mathrm{MA}=$ right motor area; $\mathrm{r}-\mathrm{S} / \mathrm{ITG}=$ right superior/inferior temporal gyrus; l-V2 $=$ left visual area; l-pFC $=$ left pFC; r-pFC = right pFC. (B) Time courses of relevant regions for short (top) and long (bottom) intervals. The $x$ axis represents time, and the $y$ axis is the mean across subjects of the absolute values of $Z$ scores. Blue and red lines correspond to temporal and neutral conditions, respectively.

high-resolution electrophysiological data combined to the application of a distributed source reconstruction modeling (Michel \& Murray, 2012) allowed to precisely depict the spatiotemporal neurodynamics for E-TP and I-TP as well as their interaction. These findings are separately discussed in the below paragraphs.

\section{Electrophysiological Signatures of E-TP}

Concerning E-TP, we identified distinct ERP configurations depending on the temporal range (short or long SOA) considered. When considering short SOAs, the main finding resulted in a prevalently left central and posterior ERP activity, namely a CNV, which was larger following temporal versus neutral cues. This modulation indicates that few hundred milliseconds after temporal cues, participants were able to implement top-down attentional modulation resulting in higher motor preparation and finally faster responses to targets. This finding is consistent with the study by Miniussi et al. (1999), which reported larger left central and posterior CNV activity for temporally valid compared with invalid cues. In support of this account, we observed a significant correlation between RTs and CNV amplitude in left centro-posterior scalp-ROIs, meaning that the larger participants' CNVs were, the faster they reacted to targets.

Concerning long SOAs, we still found an ERP pattern characterized by a central and posterior CNV preceding target onset. However, in this case the CNV was neither affected by the temporal information provided by the cue nor it significantly correlated with RTs. These data are completely in line with the absence of behavioral dif- ferences in RTs for the T-Long and N-Long conditions. Remarkably, we found two additional ERP components characterizing long trials that are an ESN and a LSP, both featured by larger amplitudes following neutral than temporal cues. The ESN was larger at left frontocentral sites for neutral long than temporal long trials. The timing and the topography of such components (see Figure 4) can likely reflect an early recruitment of motor areas controlling the right hand, the activation of which is delayed in the temporal condition. In this case, participants may have strategically postponed motor preparation. On the other hand, LSP displayed different scalp topography and morphology, being maximal at frontal scalp-ROIs, suggesting a nonmotor role of this component. The absence of external informative cues prompted a sort of temporal "uncertainty" context that forced participants to implement an internal source of TP and required a continuous updating of the conditional probability of target onset as a function of elapsing time. The morphological pattern of LSP would reflect the progressive engagement of frontal cortical areas over time and may convincingly explain why participants were overall faster at long than short intervals, regardless of the additional presence of external orienting mechanisms. In favor of this claim, we found that LSP significantly correlated with participants' performance in neutral long trials, with larger amplitudes predicting shorter RTs.

\section{Electrophysiological Signatures of I-TP}

Concerning electrophysiological signatures of I-TP, we did not find cue-related differences in electrical potentials 
between the N-Short and N-Long conditions. This was an expected result in this case, because target occurrence was actually unpredictable until an interval as long as the short SOA (1100 msec) elapsed. Hence, there was no reason to observe ERP differences within the first 1100 msec after cue offset (i.e., in T1, T2, and T3 bins). Nevertheless, once participants realized that the target did not arrive at the end of the short interval, the initial uncertainty about target occurrence turned into absolute temporal certainty, given the "aging" distribution of target probability, leading to a higher recruitment of attentional and motor resources over time.

The comparison across different temporal bins between the short and long conditions (i.e., T1 vs. T3, T2 vs. T4, and T3 vs. T6) yielded higher CNV and LSP components in the long condition for all scalp-ROI, with later bins showing larger voltages than the earlier ones. Such electrophysiological results clearly fit behavioral evidence, which shows that participants were faster for N-Long than N-Short trials.

\section{Interaction between E-TP and I-TP}

The comparison between T-Short and T-Long (E/I-TP condition) specifically allowed to investigate the interaction between E-TP and I-TP, as in this case, participants' behavioral and neural correlates were shaped by both the presence of external cues as well as by a time-based internal mechanism, both inducing temporal predictability. When comparing the mean ERP activity immediately after cue offset (T1, T2, and T3), CNV was larger for the T-Short compared with that for the T-Long condition. This could be explained by the fact that when a relevant event is predicted to occur within a short time (e.g., T-Short condition), an immediate recruitment of neural resources is needed to optimally prepare for it. However, when an event is predicted to occur later (e.g., T-Long condition), cortical resources are initially spared but maximally employed in a delayed temporal window. Specifically, by virtue of the fact that the probability of target occurrence in the T-Long condition was set up at $100 \%$ of validity, participants could allocate maximum neural resources in the final part of the SOA interval. This was confirmed by ERP data showing larger amplitudes for the T-Long compared with that for the T-Short condition when comparing the late portion of the CNV between conditions (i.e., T3 vs. T6 bins for short and long SOA, respectively). This difference may be reasonably explained considering that CNV reflects the subjective expectancy of a relevant event (Van Rijn et al., 2011), a process only indirectly based on temporal unfolding rather than an objective accumulation of temporal information per se (Macar \& Vidal, 2003). The CNV activity was larger at centro-posterior CNV sites for the late (i.e., T4, T5, and T6) than the early (T1, T2, and T3) temporal bins. As a behavioral correlate of this, participants were faster for long than for short intervals. This result further corrobo- rated the hypothesis that I-TP strongly influences behavior even in the presence of external orienting mechanisms. Notably, the absence of LSP modulations in the I-TP may be accounted for by the fact that participants did not necessarily need to update target onset probability because they could always exploit a temporal certainty about target onset.

\section{Distinct and Common Spatiotemporal Neural Networks of E-TP and I-TP}

In line with the different scalp distribution of CNV (central and posterior) and LSP (anterior), the cortical source reconstruction identified distinct neural networks involved in E-TP and I-TP. More specifically, when participants could predict a short-delayed target, they engaged a predominantly left sensorimotor cortical network. This mostly included parietal and motor areas (i.e., premotor, supplementary, and primary motor). However, when target onset was delayed even further, an additional increase of bilateral prefrontal activation emerged. As a notable finding, a prefrontal engagement was found independent of the coactivation of a left sensorimotor circuit instantiated by external TP. In other words, pFC showed increasing activity with SOA lengthening, both with (T-Long condition) and without (N-Long condition) the activation of left parietal-premotor areas. In line with behavioral evidence of (i) overall faster RTs for long compared with short SOAs and (ii) no RT differences for T-Long versus N-Long SOA, this finding may suggest that $\mathrm{pFC}$ plays a crucial role in updating target onset predictability over time, so that participants can be more prepared and faster in detecting targets after long intervals.

Although keeping into account the limitations of EEG in terms of functional localization, it is important to outline that our findings are consistent with those of previous neuroimaging evidence. We confirmed previous studies locating the TO effect (a mechanism based on exploiting external cues to generate TP) in a left sensorimotor circuit, where 1-IPC and 1-PMA areas play a crucial role (Coull et al., 2000, 2013; Cotti et al., 2011; Davranche et al., 2011; Cui et al., 2009; Macar et al., 2006; Field \& Wann, 2005; Dreher et al., 2002; Sakai et al., 2000). This neural network has been claimed to be involved in topdown allocation of attentional and motor resources over time. On the other hand, our data support previous finding claiming a central role of pFC in updating the conditional probability of stimulus occurrence over time (Vallesi, 2010; Vallesi et al., 2009; Vallesi, Mussoni, et al., 2007; Vallesi, Shallice, et al., 2007). Taken together, these data support the account that TP is established in left parietalpremotor action circuits but are monitored and potentially updated online as a function of time-in-passing, by the pFC (Coull, 2009).

As an added value of our study, the application of a source modeling on a high-density EEG array allowed, on the one hand, to identify the networks differently 
involved in different types of TP; on the other hand, the high-temporal resolution offered by EEG helped us in depicting the temporal dynamics of the interplay between such networks. This provided a complementary picture to fMRI data to better understand the functional mechanism of TP. As a main finding, we showed that E-TP for shortly expected targets engaged a left sensorimotor network just few hundred milliseconds after cue onset. This network remained recruited along all SOA and reached the maximum activation just before target onset. When target onset was predicted but longer delayed in time (T-Long), the same sensorimotor circuit showed a later activation, mirroring the ERP pattern on the scalp. This means that both attentional and motor resources allocations were delayed in time when participants knew in advance that they had more time for preparing to act. The time course of the cortical ROI depicted a steeper activation of 1-IPC, 1-PMA, and SMA after the first SOA was overcome. Overall, these data suggest that (i) E-TP shapes the allocation of attentional and motor resources over time in a top-down manner as soon as participants start preparing for an action considered as impending and (ii) attentional and motor resources conveyed by E-TP are strategically distributed over time. In contrast, when target onset is unpredictable, the first region showing significant activity is the r-pFC, suggesting that an updating of the conditional probability of target onset over time is instantiated as early as that when participants start waiting for a temporally uncertain event. Once participants realized that the target did not arrive after a short interval, updating the a posteriori conditional probability of it coming at long SOA was engaged; this process relied on a steeper r-pFC time course, probably translating at the scalp level into a steeper positive slow activity (LSP).

An interesting issue concerns the role of the SMA, which was activated more for E-TP than I-TP, leading to some speculations. Previous studies (Macar et al., 2006; Macar \& Vidal, 2003) assumed that SMA (and the CNV as its scalp ERP projection) could potentially reflect an index of accumulated time acting as a core substrate of the internal clock model (Gibbon, Church, \& Meck, 1984). This view assumes that the neural activity mirrored by $\mathrm{CNV}$ reflects the unfolding of time by itself. Alternatively, a more recent interpretation (Kononowicz \& van Rijn, 2011, 2014; Mento, 2013; Mento et al., 2013; Van Rijn et al., 2011) posits that SMA/CNV may more reliably subserve temporal expectation rather than time unfolding by itself. Our data do not allow disentangling between these two alternative accounts in that (i) we used a task purposely designed to investigate TP rather than timing per se. Therefore, it is difficult to reliably state whether time is measured here as a function of expected rather than elapsed time; (ii) participants had to perform a speeded detection task, which notoriously implies a strong motor preparation, and the engagement of a wide motor circuit including SMA (Mento, 2013). In this light, SMA may be more activated as a simple consequence of a higher motor preparation for temporally than neutrally cued targets. Hence, further studies are needed to specifically address the role of SMA in TP.

\section{General Conclusions}

This study provides new evidence that the capacity to generate TP is a flexible and dynamic brain function that can be driven according to what kind of information is made available from the environmental context. Depending on the internal or external nature of this information, distinct spatiotemporal neurodynamics are instantiated with the same final goal of reducing uncertainty about future events and to save precious resources to be employed only when actually needed. External signals allow to orient attention and to prepare motor reaction toward events occurring at predictable points in time, resulting in an early engagement of neural resources translating into negative mounting ERP activity. This activity corresponds to a centro-posterior CNV and is generated by a left sensorimotor circuit including parietal and premotor/motor areas. On the other side, in the absence of external temporal hints, occurrence of events can only be predicted by exploiting an internal mechanism intrinsically tied to the passing of time itself. Electrophysiologically, this mechanism translates into the additional elicitation of a positive increasing ERP activity corresponding to a frontal LSP reflecting the progressive engagement of prefrontal areas over time.

This evidence can be well contextualized in a more general theoretical perspective conceiving the brain as a sort of "predictive machine" whose main functional role is to generate prediction of events and to reduce uncertainty about the future (Clark, 2013; Friston, 2010). Although the rules governing the ability of generating TP are still far to be completely understood, our study sheds some light on the neurodynamics underlying this important and complex ability.

\section{Acknowledgments}

The authors kindly thank Dr. Irene Borziello for helping in collecting data and Dr. Pietro Scatturin for laboratory technical support. This work was supported by the grant "Progetto Giovani Studiosi" from the University of Padua (Prot. 1682/2012) to G. M. A. V. is funded by the ERC Starting grant LEX-MEA (GA 313692). P. S. is funded by the Bial Foundation (GA 84/2012).

Reprint requests should be sent to Giovanni Mento, General Psychology, University of Padua, Via Venezia 8, 35131 Padua, Italy, or via e-mail: giovanni.mento@unipd.it.

\section{REFERENCES}

Barnes, R., \& Jones, M. R. (2000). Expectancy, attention, and time. Cognitive Psychology, 41, 254-311.

Bischoff-Grethe, A., Martin, M., Mao, H., \& Berns, G. S. (2001). The context of uncertainty modulates the subcortical response 
to predictability. Journal of Cognitive Neuroscience, 13, 986-993.

Capizzi, M., Correa, A., \& Sanabria, D. (2013). Temporal orienting of attention is interfered by concurrent working memory updating. Neuropsychologia, 51, 326-339.

Clark, A. (2013). Whatever next? Predictive brains, situated agents, and the future of cognitive science. The Behavioral and Brain Sciences, 36, 181-204.

Correa, A., Lupiáñez, J., \& Tudela, P. (2006). The attentional mechanism of temporal orienting: Determinants and attributes. Experimental Brain Research. Experimentelle Hirnforschung. Expérimentation Cérébrale, 169, 58-68.

Correa, A., \& Nobre, A. C. (2008). Neural modulation by regularity and passage of time. Journal of Neurophysiology, 100, 1649-1655.

Cotti, J., Rohenkohl, G., Stokes, M., Nobre, A. C., \& Coull, J. T. (2011). Functionally dissociating temporal and motor components of response preparation in left intraparietal sulcus. Neuroimage, 54, 1221-1230.

Coull, J. T. (2009). Neural substrates of mounting temporal expectation. PLoS Biology, 7, e1000166.

Coull, J. T., Davranche, K., Nazarian, B., \& Vidal, F. (2013). Functional anatomy of timing differs for production versus prediction of time intervals. Neuropsychologia, 51, 309-319.

Coull, J. T., Frith, C. D., Büchel, C., \& Nobre, A. C. (2000). Orienting attention in time: Behavioural and neuroanatomical distinction between exogenous and endogenous shifts. Neuropsychologia, 38, 808-819.

Coull, J. T., \& Nobre, A. C. (1998). Where and when to pay attention: The neural systems for directing attention to spatial locations and to time intervals as revealed by both PET and fMRI. The Journal of Neuroscience: The Official Journal of the Society for Neuroscience, 18, 7426-7435.

Cui, X., Stetson, C., Montague, P. R., \& Eagleman, D. M. (2009). Ready...go: Amplitude of the fMRI signal encodes expectation of cue arrival time. PLoS Biology, 7, e1000167.

Davranche, K., Nazarian, B., Vidal, F., \& Coull, J. (2011). Orienting attention in time activates left intraparietal sulcus for both perceptual and motor task goals. Journal of Cognitive Neuroscience, 23, 3318-3330.

Drazin, D. H. (1961). Effects of foreperiod, foreperiod variability, and probability of stimulus occurrence on simple reaction time. Journal of Experimental Psychology: General, 62, 43-50.

Dreher, J.-C., Koechlin, E., Ali, S. O., \& Grafman, J. (2002). The roles of timing and task order during task switching. Neuroimage, 17, 95-109.

Field, D. T., \& Wann, J. P. (2005). Perceiving time to collision activates the sensorimotor cortex. Current Biology, 15, 1-6.

Friston, K. (2010). The free-energy principle: A unified brain theory? Nature Reviews Neuroscience, 11, 127-138.

Gibbon, J., Church, R. M., \& Meck, W. H. (1984). Scalar timing in memory. Annals of the New York Academy of Sciences, $423,52-77$.

Gramfort, A., Papadopoulo, T., Olivi, E., \& Clerc, M. (2010). OpenMEEG: Opensource software for quasistatic bioelectromagnetics. Biomedical Engineering Online, 9, 45.

Gratton, G., Coles, M., \& Donchin, E. (1983). A new method for off-line removal of ocular artifact. Electroencephalography and Clinical Neurophysiology, 55, 468-484.

Henson, R. N., Shallice, T., \& Dolan, R. J. (1999). Right prefrontal cortex and episodic memory retrieval: A functional MRI test of the monitoring hypothesis. Brain: A Journal of Neurology, 122, 1367-1381.
Karlin, L. (1958). Reaction time as a function of foreperiod duration and variability. Journal of Experimental Psychology: General, 58, 185-191.

Kononowicz, T. W., \& van Rijn, H. (2011). Slow potentials in time estimation: The role of temporal accumulation and habituation. Frontiers in Integrative Neuroscience, 5, 48

Kononowicz, T. W., \& van Rijn, H. (2014). Decoupling interval timing and climbing neural activity: A dissociation between CNV and N1P2 amplitudes. The Journal of Neuroscience: The Official Journal of the Society for Neuroscience, 34, 2931-2939.

Los, S. A., \& Heslenfeld, D. J. (2005). Intentional and unintentional contributions to nonspecific preparation: Electrophysiological evidence. Journal of Experimental Psychology: General, 134, 52-72.

Luce, R. D. (1986). Response times: Their role in inferring elementary mental organization. New York: Oxford University Press

Macar, F., Coull, J., \& Vidal, F. (2006). The supplementary motor area in motor and perceptual time processing: fMRI studies. Cognitive Processing, 7, 89-94.

Macar, F., \& Vidal, F. (2003). The CNV peak: An index of decision making and temporal memory. Psychophysiology, 40, 950-954.

Mento, G. (2013). The passive CNV: Carving out the contribution of task-related processes to expectancy. Frontiers in Human Neuroscience, 7, 827.

Mento, G., Tarantino, V., Sarlo, M., \& Bisiacchi, P. S. (2013). Automatic temporal expectancy: A high-density event-related potential study. PloS One, 8, e62896.

Michel, C. M., \& Murray, M. M. (2012). Towards the utilization of EEG as a brain imaging tool. Neuroimage, 61, 371-385.

Miniussi, C., Wilding, E. L., Coull, J. T., \& Nobre, A. C. (1999). Orienting attention in time. Modulation of brain potentials. Brain: A Journal of Neurology, 122, 1507-1518.

Niemi, P., \& Näätänen, R. (1981). Foreperiod and simple reaction time. Psychological Bulletin, 89, 133-162.

Nobre, A., Correa, A., \& Coull, J. (2007). The hazards of time. Current Opinion in Neurobiology, 17, 465-470.

Nobre, A. C. (2001). Orienting attention to instants in time. Neuropsychologia, 39, 1317-1328.

Petrides, M. (2005). Lateral prefrontal cortex: Architectonic and functional organization. Philosophical Transactions of the Royal Society of London, Series B, Biological Sciences, 360, 781-795.

Sakai, K., Hikosaka, O., Takino, R., Miyauchi, S., Nielsen, M., \& Tamada, T. (2000). What and when: Parallel and convergent processing in motor control. The Journal of Neuroscience: The Official Journal of the Society for Neuroscience, 20, 2691-2700

Stuss, D. T., Alexander, M. P., Shallice, T., Picton, T. W., Binns, M. A., Macdonald, R., et al. (2005). Multiple frontal systems controlling response speed. Neuropsychologia, 43, 396-417.

Tadel, F., Baillet, S., Mosher, J. C., Pantazis, D., \& Leahy, R. M. (2011). Brainstorm: A user-friendly application for MEG/EEG analysis. Computational Intelligence and Neuroscience, 2011, 879716

Trillenberg, P., Verleger, R., Wascher, E., Wauschkuhn, B., \& Wessel, K. (2000). CNV and temporal uncertainty with "ageing" and "non-ageing." Clinical Neurophysiology, 111, 1216-1226.

Tzourio-Mazoyer, N., Landeau, B., Papathanassiou, D., Crivello, F., Etard, O., Delcroix, N., et al. (2002). Automated anatomical labeling of activations in SPM using a macroscopic anatomical parcellation of the MNI MRI single subject brain. Neuroimage, 15, 273-289. 
Vallesi, A. (2010). Neuro-anatomical substrates of foreperiod effects. In J. T. Coull \& A. C. Nobre (Eds.), Attention and time (pp. 303-316). Oxford, UK: Oxford University Press.

Vallesi, A., McIntosh, A. R., Shallice, T., \& Stuss, D. T. (2009). When time shapes behavior: fMRI evidence of brain correlates of temporal monitoring. Journal of Cognitive Neuroscience, 21, 1116-1126.

Vallesi, A., Mussoni, A., Mondani, M., Budai, R., Skrap, M., Shallice, T., et al. (2007). The neural basis of temporal preparation: Insights from brain tumor patients. Neuropsychologia, 45, 1-29.
Vallesi, A., Shallice, T., \& Walsh, V. (2007). Role of the prefrontal cortex in the foreperiod effect: TMS evidence for dual mechanisms in temporal preparation. Cerebral Cortex (New York, N.Y.: 1991), 17, 466-474.

Van Rijn, H., Kononowicz, T. W., Meck, W. H., Ng, K. K., \& Penney, T. B. (2011). Contingent negative variation and its relation to time estimation: A theoretical evaluation. Frontiers in Integrative Neuroscience, 5, 91.

Wiener, M., Turkeltaub, P., \& Coslett, H. B. (2010). The image of time: A voxel-wise meta-analysis. Neuroimage, 49, 1728-1740.

Woodrow, H. (1914). The measurement of attention. The Psychological Monographs, 17, 1-158. 